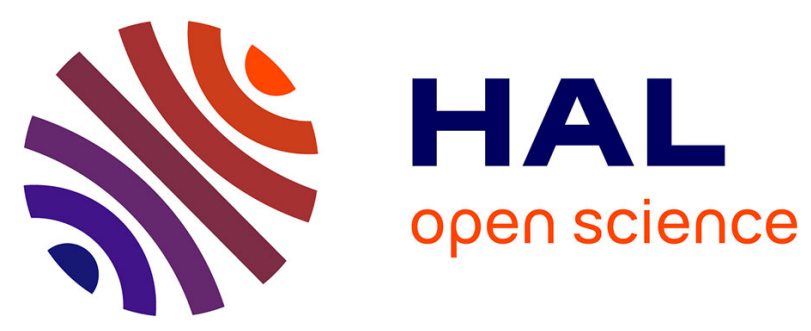

\title{
Experimental validation of transient spectral finite element simulation tools dedicated to guided wave based structural health monitoring
}

Olivier Mesnil, Arnaud Recoquillay, Tom Druet, Valentin Serey, Huu Tinh Hoang, Alexandre Imperiale, Edouard Demaldent

\section{To cite this version:}

Olivier Mesnil, Arnaud Recoquillay, Tom Druet, Valentin Serey, Huu Tinh Hoang, et al.. Experimental validation of transient spectral finite element simulation tools dedicated to guided wave based structural health monitoring. Journal of Nondestructive Evaluation, Diagnostics and Prognostics of Engineering Systems, In press, 10.1115/1.4050708 . hal-03187419

\author{
HAL Id: hal-03187419 \\ https://hal.science/hal-03187419
}

Submitted on 19 Apr 2021

HAL is a multi-disciplinary open access archive for the deposit and dissemination of scientific research documents, whether they are published or not. The documents may come from teaching and research institutions in France or abroad, or from public or private research centers.
L'archive ouverte pluridisciplinaire HAL, est destinée au dépôt et à la diffusion de documents scientifiques de niveau recherche, publiés ou non, émanant des établissements d'enseignement et de recherche français ou étrangers, des laboratoires publics ou privés. 


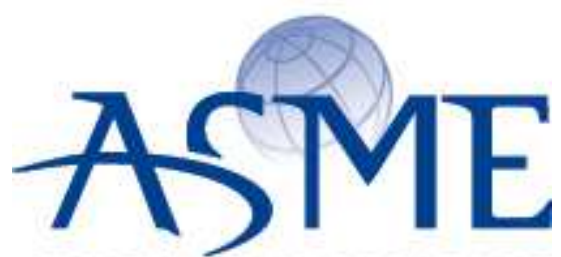

SETTING THE STANDARD

\section{American Society of Mechanical Engineers}

\section{ASME Accepted Manuscript Repository}

\section{Institutional Repository Cover Sheet}

ASME Paper Title: Experimental Validation of Transient Spectral Finite Element Simulation Tools Dedicated to Guided Wave-Based Structural Health Monitoring

Olivier Mesnil , Arnaud Recoquillay, Tom Druet, Valentin Serey, Huu Tinh Hoang , Alexandre

Authors: Imperiale, Edouard Demaldent

ASME Journal Title: ASME J Nondestructive Evaluation

Volume/Issue $4(4)$ Date of Publication (VOR* Online) April 19, 2021

https://asmedigitalcollection.asme.org/nondestructive/article/4/4/041003/1105952/I ASME Digital Collection URL: $\underline{\text { Validation-of-Transient-Spectral }}$

DOI:

*VOR (version of record) 


\title{
Experimental validation of transient spectral finite element simulation tools dedicated to guided wave based structural health monitoring
}

\author{
Olivier Mesnil*, Arnaud Recoquillay, Tom Druet, Valentin Serey, \\ Huu Tinh Hoang, Alexandre Imperiale, Edouard Demaldent \\ Université Paris-Saclay, CEA, List, \\ F-91120, Palaiseau, France
}

In Guided Wave Structural Health Monitoring (GW-SHM), a strong need for reliable and fast simulation tools has been expressed throughout the literature in order to optimize SHM systems or demonstrate performance. Even though guided wave simulations can be conducted with most finite elements software packages, computational and hardware costs are always prohibitive for large simulation campaigns. A novel SHM module has been recently added to the CIVA software and relies on unassembled high order finite elements to overcome these limitations. This paper focuses on the thorough validation of CIVA for SHM to identify the limits of the models. After introducing the key elements of the CIVA SHM solution, a first validation is presented on a stainless steel pipe representative of the oil and gas industry. Second, validation is conducted on a composite panel with and without stiffener representative of some structures in the aerospace industry. Results show a good match between the experimental and simulated datasets, but only if the input parameters are fully determined prior to the simulations.

\section{Introduction}

Guided Wave Structural Health Monitoring (GW-SHM) relies on the permanent integration of sensors to monitor the health of a structure over time. Despite a large and convincing literature exhibiting various proofs of concepts, GWSHM hasn't reached all of its expected objectives: Primarily mainstream preventive maintenance based on real time health assessment, but also structural life extension. Among the limitations to deployment of GW-SHM systems is a systematic and rigorous system optimization and performance demonstration framework.

The uses of simulations in GW-SHM are various. First and foremost, simulations have been conducted to analyze and explain experimental results, or simply to develop processing techniques [1-3] prior to experimental deployment. Second, simulations in GW-SHM are foreseen to play a significant role in Model Assisted Probability of Detection

${ }^{*}$ Corresponding author: Olivier.mesnil@cea.fr
(MAPOD) approach to avoid prohibitively expensive experimental campaigns. More recently, the rise of artificial intelligence has created a need of large databases to train deep learning algorithms to enable efficient and robust diagnostic [4]. However deep learning requires very large datasets which, in general, cannot be obtained by experiments, therefore require large simulation campaigns.

Due to the high number of influencing parameters in GW-SHM, conducting such analysis using exclusively experimental data is prohibitively expensive and for this reason, simulation frameworks have been proposed to simulate guided wave propagation in large structures. Semi-analytical methods have been widely studied in the literature [5-7] for the propagation of GWs in infinite structures of finite cross sections. In a time-harmonic regime, such techniques have then been coupled to finite elements to create hybrid solvers able to describe large structures with defects [8-11] but remain somewhat limited in terms of geometry. On the other hand and for most papers in the literature dealing with this topic, full $3 \mathrm{~d}$ transient finite element solvers have been used in GW propagation simulations. However due to small element size and time stepping requirements intrinsic to $\mathrm{GW}$, finite elements simulations are computationally costly. A benchmark of the most common finite element software platforms was conducted in 2018 on a single composite panel [12] of relatively small dimensions: $120 \times 60 \times 1 \mathrm{~mm}$ with 8 plies. The authors compared computational times from 20 hours to up to one week for a single simulation at $300 \mathrm{kHz}$ of central frequency, with powerful computer hardware and CPU architectures.

Recently, and thanks to the development of affordable and efficient computing hardware, especially GPUs, novel GW simulation techniques have emerged relying on intensive parallelization of mesh groups [13-16]. If these methods have shown excellent performance, with improvements of 2 to 3 orders of magnitude with respect to traditional finite element methods on CPU, they have three limitations. First, they require a dedicated and expensive hardware, which limits the capacity to run multiple simulations in parallel as GPU 
computer clusters are not as easily available as CPU clusters. Second, the maximal size of the configurations covered by GPU solvers is limited by the memory available on the GPU. For example in the case of the benchmark previously mentioned [12] about 40 Go of memory were required to run on a GPU [16]. Finally, if GPU computations are very fast, communication between the nodes is relatively slow which leads to a significant slowing of the simulation during data extraction, greatly diminishing the gain associated to GPU for simulations when large data extraction is required.

In a different paradigm, Spectral Finite Element methods (SFEM) have been proposed to significantly reduce the computational burden through a reduction of the number of degrees of freedom and a more efficient computation at each time step [17-19], which is the core of the solver presented in this article. More details on the solver will be presented in Section 2, but for comparison purposes, its resolution of the case of the 2019 benchmark [20] took 12 minutes (for approximately $10^{6}$ degrees of freedom) on a regular desktop computer and a near negligible memory requirement, enabling parametric studies on realistic time spans with close to no hardware limitations. This solver was recently integrated into the software platform CIVA for large dissemination toward users by the EXTENDE company [21].

Regardless of the simulation procedure, a simulation is useful only if it has been thoroughly validated. Compared to finite elements of order one or two, SFEM have received far less interest (see however [17]) in terms of validation for guided wave propagation. For this reason, the goal of this paper is to validate the simulation obtained with CIVA for the simulation of GW propagation for two applications of interest in GW-SHM: the monitoring of metallic pipes in the pipeline industry and the monitoring of large composite structures in aeronautics. First for the pipe application, data acquired by the authors on a stainless steel pipe was used to validate the simulations. For the composite case however, the elastic properties of most composite panels being afflicted of large uncertainties, the online database openguidedwaves.de [22] was used. Indeed, the authors of this database have been through a lengthy calibration procedure of the elastic properties of the composite panels under study, which is essential to match experimental and simulated data on composite. The main contribution of this work lies in the combination, integration and validation of the spectral finite element techniques to obtain an efficient simulation tool, hence enabling parametric analysis and probability of detection studies.

The outline of the paper is the following: first in Section 2, the main modelling assumptions of CIVA for GW-SHM will be presented. Validation is presented for three different use-cases in Sections 3 to 6: first a stainless steel pipe, second a flat composite panel and finally a stiffened composite panel. Conclusions are presented in Section 7.

\section{CIVA solver for GW-SHM simulations}

This section describes the simulation strategy used in the GW-SHM module of CIVA. In order to obtain fast and accu- rate simulations with low memory requirements, an explicit finite difference scheme in the time domain is coupled with high order spectral finite elements in space. The main difficulty is then the meshing process to allow the use of such high order elements in a user friendly software. First, the overall strategy using macro-elements is described before giving the main characteristics of spectral finite elements. Finally, the specifics of the modelling of piezoelectric transducers are given.

\subsection{Unassembled spectral finite elements}

The first step of the simulation process is the definition of a macro-scale mesh of the geometry. All geometries under consideration, that is plates and pipes with added elements (such as stiffeners), can be decomposed into a collection of macro-elements, which are unit cubes up to a determined transform (see Figure 1). The order of the geometric transform can be high to take into account curvature, for example in the case of a pipe, a curved plate or a stiffener. The macroelements are then subdiscretized at the wavelength scale with respect to the stacking sequence in the case of stratified media, e.g. composites structures. This subdiscretization is parametrically defined, thus having negligible memory footprint, and optimal data structures for multi-threaded finite element operations.

It is then easy to define spectral finite elements on the reference unit cube and use the transform to take the real geometry into account (see [19] for more details): indeed, in the unit cube, 3D - spectral finite elements are defined as the tensorial product of 1D - spectral finite elements. These 1D elements are Lagrange polynomials, as in classic low-order finite elements, but using Gauss-Lobatto nodes, defined at order $n$ as the roots of $\left(1-x^{2}\right) P_{n}(x)=0$, with $P_{n}$ the Legendre polynomial of order $n$, instead of equi-spaced nodes. The use of Gauss-Lobatto nodes induces that these elements can be efficiently defined for any given order. The main advantage of spectral finite elements in our case is then the high-order interpolation of the solution, allowing a reduced number of degrees of freedom, as well as mass-lumping, drastically reducing the inversion cost in the computation as the mass matrix, which is inverted at every time step, is diagonal. Its inversion is then straightforward and there is no need to assemble it.

In the CIVA for SHM module, the time marching algorithm of the leapfrog time scheme is used. In this context, since the mass matrix is diagonal (at any order), the main computational cost resides in performing the product between the stiffness matrix with the finite element vectors. This operation is performed element-by-element, in parallel, and on-the-fly ("unassembled" operations). By doing so, no assembling of the global stiffness matrix is required, thus significantly reducing the memory footprint of the solver.

Spectral finite elements require hexahedral meshes, and producing such meshes for a given description of the specimen is known to be a major challenge in computational geometry. Hence, the main drawback of this strategy is the limitation on the available configurations. To circumvent this 


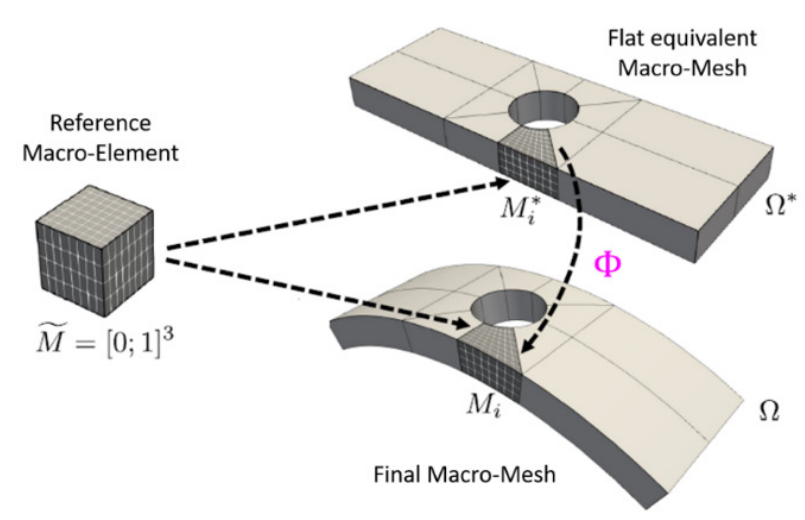

Fig. 1: Scheme of the macro-element strategy, see [19] for more details.

difficulty we resort to an analytic parametrization of each configuration. A significant number of geometries are already available in the CIVA SHM module, and new ones will be added. Note that this is not a limitation of the SFEM code but a restriction of the GUI of CIVA SHM, which does not require a priori expertise in finite element simulation. In addition, this restriction to parametric configurations facilitates the conduct of sensitivity studies in the CIVA framework.

The meshing procedure is performed in the following way: first guided modes are computed on the frequency range of the excitation signal and the smallest wavelength of the computation is deduced from it. More precisely, the wavelengths of the propagating modes are computed thanks to a Semi-Analytical Finite Element method (SAFE) [5] on the frequency range of the excitation signal. Plate modes are considered in all cases. In the special case of a pipe, circumferential modes, taking into account the bending of the plate to describe the pipe, are also computed. The meshing is then done to achieve a given number of points per wavelength, this number depends on the order of the used elements. Note that between 6 to 10 points per wavelength are ensured, which is sufficient to obtain a good approximation of the solution in the case of SFEM [23]. This value is smaller than the usual "rule of thumb" of 15 to 20 points per wavelength for low-order methods. Finally, the module shall shortly include damping modelling based on [24], but all results presented in this paper are without damping.

\subsection{Piezoelectric modelling}

In order to further reduce the computational cost of a configuration, a simplified model is used for the piezoelectric transducers. The source is modelled as a surface load on the geometry whereas the measure is defined as an integral over the perimeter of the sensor. More precisely, thin piezoelectric disks are considered in this article, which are modelled using the model first introduced in [25]: the load is axisymmetric and applied on the circumference of the transducer, in the radial direction. The measurement is the integral on this circumference of the radial displacement. It is well known that for low frequencies on metallic plates, the results of this

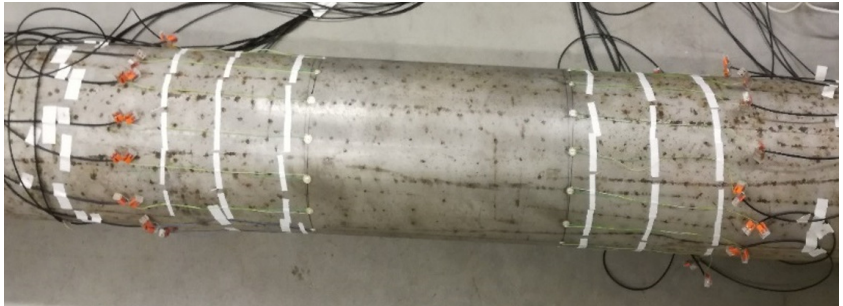

Fig. 2: Experimental setup. Picture of the pipe with the bounded piezoelectric transducers.

model are in good agreements with the experiment. Its indepth validity in the context of anisotropic structures will be the topic of a follow-up publication. Note that it is needed to define a surface load in the Finite Element computation, whereas this model gives a load over a line. To circumvent this issue, a small ring is defined at the edge of the sensor, over which the integration takes place.

It should be noted that this model has a drawback: to limit as much as possible the number of degrees of freedom used in the mesh, and because the sensors are usually rather small with respect to the wavelengths, the transducers may be poorly approximated as only a few nodes are included in its support. To avoid this, a special care is given to the sensors mesh and a specific mesh pattern is defined for the corresponding macro-elements, which induces smaller time steps as the corresponding elements are small. Indeed, for a given material and a fixed time-window, the overall number of time steps depends on the ratio of the minimum element size over the maximum element size, which is driven by the wavelength. Indeed, the time step is given by the CFL (Courant Friedrichs Lewy) condition: $\Delta t \leq 2 / \sqrt{\rho\left(M^{-1} K\right)}$, where $\Delta t$ is the maximal time step, $K$ and $M$ stand for the stiffness and mass matrices, respectively, and $\rho$ for the spectral radius. While the spectral radius does depend on the ratio of the minimum over maximum element size, the minimum element size is fixed by the geometric singularities (most often the meshing pattern of the sensors, but also sometimes the defect). On the other hand, the maximum element size depends on the wavelength.

\section{Validation of wave propagation in a stainless steel pipe}

In this section are shown comparisons between experimental measurements in a welded stainless steel pipe (see figure 2) with the corresponding simulated signals.

The pipe of figure 2 is of the following geometry: outer diameter of $254.29 \mathrm{~mm}$, thickness of $2.145 \mathrm{~mm}$ and a length of $2.81 \mathrm{~m}$. Two transducer rings distanced of $40 \mathrm{~cm}$ and composed of fifteen sensors each are bounded to the structure. The sensors are piezoelectric transducers with a diameter of $8 \mathrm{~mm}$. In all acquisitions, one sensor acts as an actuator whereas all others act as receivers. The source signal is in all cases a Hanning tone burst emission of $50 \mathrm{kHz}$ generated by a waveform generator (Keysight Technologies) with a peak-to-peak amplitude of $\pm 10 \mathrm{~V}$. The signals mea- 
sured by the receivers are first filtered analogically and amplified by a low-noise preamplifier (Stanford Research Systems) with a second order high-pass filter with a cut-off frequency of $10 \mathrm{kHz}$. Then, the signals are digitized using an oscilloscope (Teledyne LeCroy) and averaged 30 times to increase the signal to noise ratio. Multiplexers allow to switch between the piezoelectric transducers. The overall acquisition process is fully automated thanks to a LabVIEW code. The experiment was conducted in CEA laboratories.

The exact material properties of the pipe are unknown, but a high frequency longitudinal bulk wave pulse echo acquisition was performed on various points of the pipe to assess its homogeneity. The measured times of flight exhibit a variation of the order of $5 \%$ of the longitudinal waves velocity along the circumference. This variation is in good agreement with standard wall thickness tolerances for welded pipes. In order to see how this variability affects the propagation, a first comparison was performed on the signals corresponding to the emitter on the first transducer ring and the receiver on the second ring, on the same axis. In a perfect pipe with no variability, these signals should be identical, up to uncertainties regarding the sensors. The fifteen experimental signals are shown in figure 3 . Note that on those signals, the experimental electromagnetic coupling can be seen around $50 \mu$ s, while the wave packets around $250 \mu$ s to $300 \mu$ s correspond to the pipe mode $\mathrm{L}(0,1)$ equivalent to $\mathrm{A} 0$ in a plate. A high variability of the phase of the signals can be seen for this mode between each measurement. For our interrogating frequency of $50 \mathrm{kHz}$, such a large phase variation cannot be due to the experimental uncertainties related to the placement of the sensors as this would imply a positioning error greater than $5 \mathrm{~mm}$. However, this phase variation is due to the variability of the geometrical and material properties along to the circumference of the pipe. On the other hand, the significant amplitude variation may be due to several factors. First, two signals of very low amplitude and one of very large amplitude compared to the others are visible. After investigation, it was observed that the signal with the smallest amplitude corresponds to the sensors bounded directly on the weld of the pipe. Indeed, as the weld has a different micro structure compared to the rest of the pipe, the wave packets are scattered for this specific sensor pair path. The two other signals (low amplitude and largest one) are the neighboring couples of sensors at a distance of about $5 \mathrm{~cm}$ from the weld. Hence, these amplitude variations seem strongly linked to the weld. Note that the arrangement is not perfectly symmetrical with respect to the weld and that a thickness irregularity, asymmetric with respect to the weld, was observed in the area and might explain the amplitude variation. Second, the amplitude variation may be due to the coupling of the sensors which is not perfectly reproducible from one sensor to another, or the variation of the sensors themselves.

Several simulations have been run with CIVA SHM for the same geometry, position of sensors and inspection frequency. A parametric study on the material properties has been performed to identify the best match among the parameters within the interval of values. More precisely, standard values for the Young modulus and the Poisson ratio of stain-

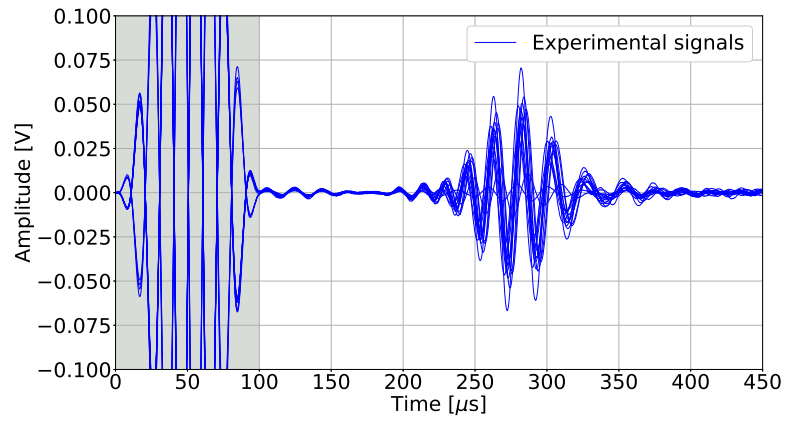

Fig. 3: Experimental signals for axial couples of sensors (emitter in the first ring and receiver in the second one in front of the emitter).

less steel, that is $197 \mathrm{GPa}$ and 0.299 (with $\rho=7890 \mathrm{~kg} \mathrm{~m}^{-3}$ ), were taken as an initial guess and a variation of the Young modulus was performed, corresponding to a $\pm 2.5 \%$ of variation of the longitudinal waves velocity. The results are presented in figure 4 . The set of experimental results is represented by a blue beam from which the three aberrant signals corresponding to the couples close to the weld are removed from the experimental set. From this result, it can be observed that the best elastic property fit is at $E=188 \mathrm{GPa}$ as represented by the green curve as it intersects quite well the experimental beam whether it is for $\mathrm{L}(0,1)(\approx \mathrm{A} 0$ in plates $)$ or for $\mathrm{L}(0,2)$ around $100 \mu \mathrm{s}(\approx \mathrm{S} 0$ in plates).

After calibration of the simulation parameters, a comparison is performed for a sensor acting as actuator on the first ring and all fifteen sensors of the second ring are acting as receivers. The results are shown in figure 5. First, it can be observed that the wave packets corresponding respectively to the direct and first helical modes of $\mathrm{L}(\mathrm{m}, 1)$ and $\mathrm{L}(\mathrm{m}, 2)$ have similar time of flights for all signals, hence the simulation of the group velocity is satisfying. Regarding the phase of the signal, a mismatch is once again observed. More precisely, the phase is close to equal for signals corresponding to sensors aligned or close to aligned along the axis of the pipe, that is the bottom signal and the top one, and the difference grows with the difference in circumferential position between the emitter and the receiver. This can be explained by the variability of thickness, which is not taken into account in the simulation.

\section{Validation of wave propagation in composite through wavefield analysis}

In this section are shown results of the validation study of a wavefield propagating in a flat composite panel.

\subsection{Experimental data}

As previously observed, a major difficulty in validating simulations is to obtain a complete and precise knowledge of the influencing parameters. As GWs are especially sensitive to many parameters, this is a significant challenge for validation in general and more specifically, for composite materi- 


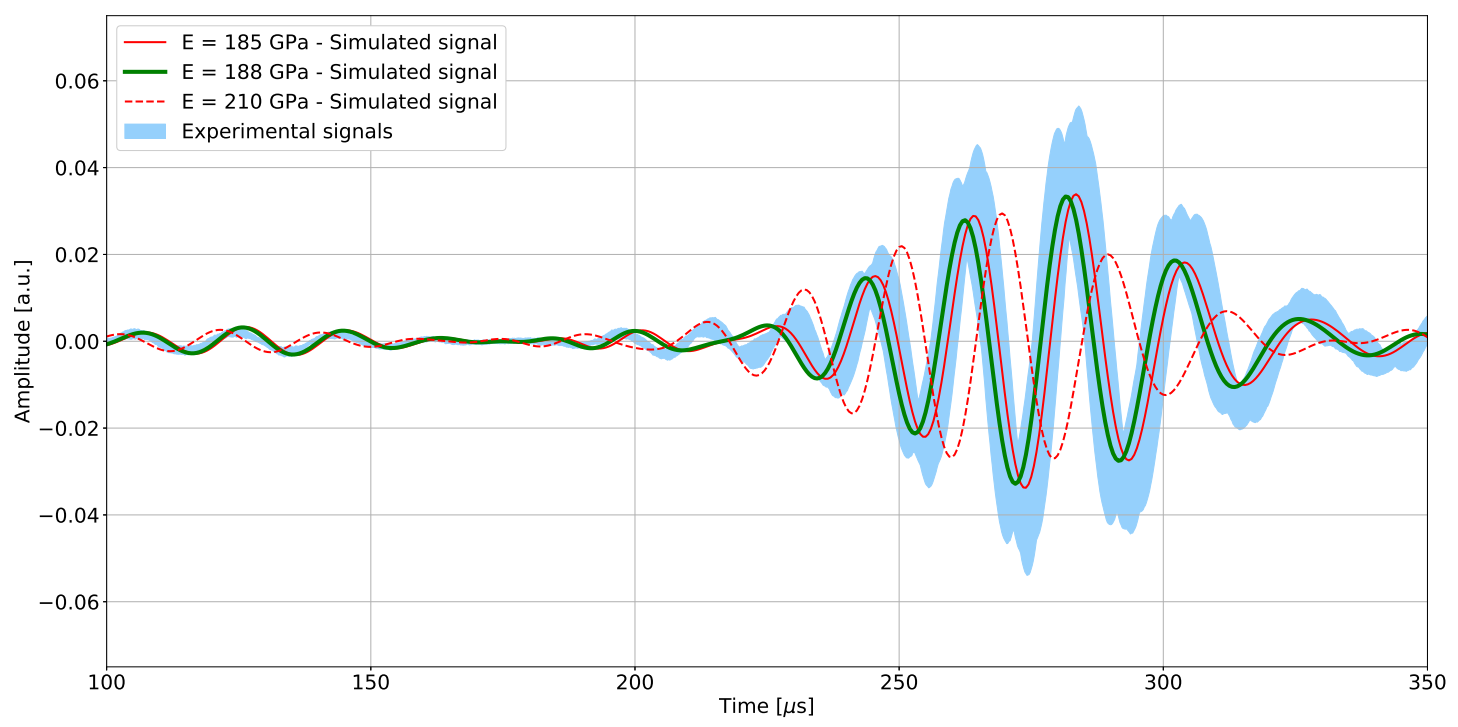

Fig. 4: Comparison between experimental signals and simulated signals for axial couples of sensors and various Young's modulus.

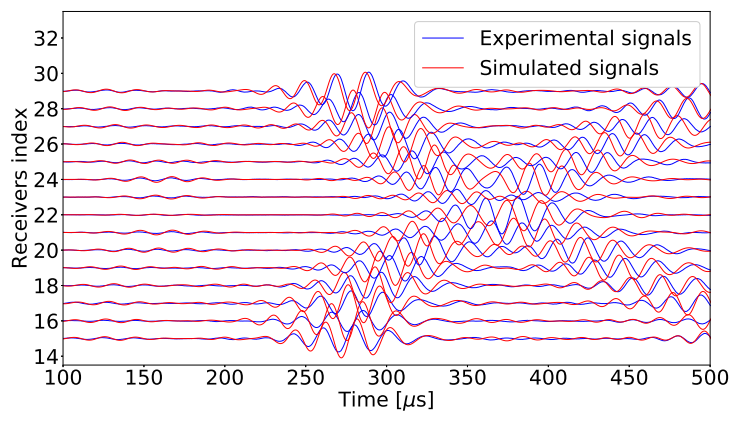

Fig. 5: Experimental signals and simulated signals comparison for a full ring of sensors with the calibrated Young's modulus.

als. Indeed, most often the elastic properties values are not precisely known as manufacturers often provide conservative values for the use of composite as load carrying structures. Moreover, elastic properties of composite structures can vary over a fairly large range of values due to manufacturing tolerances. For example, carbon fiber content in Carbon Fiber Reinforced Composite (CFRP) might vary up to $\pm 4 \%$ [26]. By a quick calculation with the rule of mixture and the Semi Analytical Finite Element technique (SAFE) [5], this roughly leads to an error in terms of the group velocity of the A0 mode up to $6 \%$ around $100 \mathrm{kHz}$, which is a far greater effect than the one induced by most defect one might want to detect in such structures. For this reason, a proper knowledge of the elastic properties of the composite is essential for validation.

In this work, the validation is conducted on data provided by the Open Guided Waves (OGW) initiative [22], as the authors went through an extensive calibration procedure of the elastic properties of the composite sample under consideration, which significantly reduces the main source of potential mismatch between simulations and experiments. This section focuses on reproducing the wavefield measured by a laser vibrometer on a plate. The main parameters of this experiment are copied here, but for more details the reader is invited to refer to [22]. The studied composite panel is a 16-layer CFRP panel of dimensions $500 \times 500 \times 2 \mathrm{~mm}^{3}$. It is instrumented by a piezoelectric actuator in its center and the propagated wavefield is measured by a 3D laser Doppler Vibrometer in one quarter of the plate on a grid with a $1 \mathrm{~mm}$ spatial step. To enhance the quality of the experimental data, a low-pass Butterworth filter of order 5 is applied to every experimental measurement with a cut-off frequency corresponding to three times the central excitation frequency.

\subsection{Simulated configurations}

The previously described configuration is replicated with the CIVA software with the elastic properties provided by OGW. The simulation is full 3D: no symmetry argument was used and each layer is modeled by a 3D solid element, resulting in 16 plies of dimensions $500 * 500 \mathrm{~mm}$. The local orientation of the anisotropy within each element is carried by the macro-mesh, which is composed of a single 3D solid element over the entire thickness (that carries the distortion of the reference material) plus a 1D description of the reference material in the stacking (elasticity coefficients, fiber orientation and thickness in a straight medium). These data are taken directly from the input parameters of the CIVA GUI.

Simulations are conducted for 5-cycle tone bursts of central frequency 50, 100, 150 and $200 \mathrm{kHz}$. Displacements are extracted from the simulations at a grid of points corresponding to the experimental laser scan. Note that in the OGW dataset, defects are added by using an attached mass, which is not a defect available in CIVA. The analysis of defective wavefield will therefore not be conducted in this section but some defect imaging will be shown in the following 
section.

\subsection{Validation results on wavefield}

The simplest qualitative validation step consists in observing the wavefield generated by CIVA and compare it to the measurements as represented in figure $6,100 \mu$ s after the $50 \mathrm{kHz}$ excitation. At first glance, the main difference between these results is the noise level, inexistent in the simulations and dominant in the in-plane components of the experiment. Due to the noisiness of the measurement of the in-plane components, the validation of the wavefield will focus on the comparison of the out-of-plane component for the remaining of this section. Note that it means that this section focuses on the A0 mode which is mainly out-of-plane, while the S0 mode, dominated by its in-plane components, will be studied in the following section. The comparison of dispersion curves between experimental and simulated data are displayed in figure 7 . Note that for each of these plots, the dispersion curve is compared to the theoretical values provided by the SAFE technique [5] and computed with CIVA. The left column displays the experimental results from 50 to $200 \mathrm{kHz}$ and the right column displays the simulated results. As expected, the simulated results are perfectly superimposed with the theoretical calculation provided by SAFE, however a small shift can be observed in the experimental data (note that the same shift can be observed in the figure 9 of [22]). As the elastic properties have been measured with relatively small confidence intervals, this mismatch between experiment and theory is most likely explained by the remaining unknowns of the plate (plate homogeneity, temperature at the time of the measurement versus the one of the calibration...).

To go further in the validation process, three arbitrary points are chosen to compare the out-of-plane components of the wavefield. The emitter is located at the coordinate $p_{0}=(250,250) \mathrm{mm}$ in figure 6 (i.e. the top right corner). The comparison points are selected at the coordinates $p_{1}=(200,200), p_{2}=(225,100)$ and $p_{3}=(100,50) \mathrm{mm}$ corresponding to the cylindrical coordinates from the actuator of $\left(62 \mathrm{~mm}, 42^{\circ}\right),\left(147 \mathrm{~mm}, 7^{\circ}\right)$ and $\left(242 \mathrm{~mm}, 36^{\circ}\right)$ respectively. The Ascan comparison for each point is shown in figures 8,9 and 10 for the frequencies 50,100 and $150 \mathrm{kHz}$ respectively. The $200 \mathrm{kHz}$ comparison is not shown as the experimental data are too noisy to conclude for this frequency. Note that all the data have been normalized to unity to focus on phase and time of flight comparison. At $50 \mathrm{kHz}$ in figure 8, an excellent envelope match is observed for the three Ascans with a small mismatch for $p_{3}$ and a phase shift for all the points. These small errors are most likely due to the mismatch observed in the dispersion curve.

At $100 \mathrm{kHz}$ in figure 9, a perfect time of flight match is observed for the three points along with the reflections from the edges observed in $p_{2}$ and $p_{3}$. The amplitude of the reflection in $p_{2}$ is not properly captured because attenuation effects are neglected in the simulations. The amplitude of the reflection in $p_{3}$ is roughly correct as it is near the edge of the plate, the wave-packets have not been attenuated much at this posi- tion, unlike in $p_{2}$. As previously, the phase does not match: in opposition for the first two points and roughly in phase for the last one. Once again, this is most likely due to the dispersion curve mismatch previously discussed. At $150 \mathrm{kHz}$ in figure 10, the experimental measurements are of too poor quality to conclude. Further analysis leads to the observation that the A0 mode is highly attenuated at these frequencies and beyond, and even the out-of-plane measurement carries very little content above the noise level. No conclusion can therefore be extracted from this data and for the same reason, the data at $200 \mathrm{kHz}$ is not represented.

\subsection{Computational performance}

Computational efficiency is essential for GW simulations in SHM as usually several simulations are conducted simultaneously or sequentially. For example in order to simulate a round-robin scan (i.e. each transducer of a network excites successively a wave while the others act as receivers), optimize SHM systems or conduct parametric studies on the variations of external parameters. As discussed in Section 2, CIVA SHM is especially efficient in terms of both CPU and memory usage as summarized in Table 1 for the 4 wavefield simulations of interest. First, it can be observed from this table that the time step remains constant for the first three frequencies, which is due to the fact that at low frequencies the smallest element is within the sensor area and is independent of the frequency. Second, the number of degrees of freedom increases roughly quadratically with the frequency, which is due to the fact that the mesh is refined only in-plane for an increase in frequency, while the out-of-plane mesh is already very fine (due to the multiple plies) and needs no further refinement. Finally, CPU time and RAM usage scale roughly linearly with the number of degrees of freedom. It can be concluded that CPU time increases quadratically with the frequency if the time step remains constant and cubically if the time step is reduced with the frequency increase.

For comparison purposes, a COMSOL Multiphysics simulation was conducted at $100 \mathrm{kHz}$ on the same 16-ply CFRP panel for $300 \mu s$. Because the memory requirements on COMSOL were larger than the available RAM, a reduced domain was considered: both width and length are reduced from 500 to $50 \mathrm{~mm}$, reducing the overall area of a factor 100 . The sensor is modelled in COMSOL with the same model as the one used in CIVA. The mesh size and time steps of COMSOL are optimized for tetrahedral finite elements of order 1. For the two iterative solvers of COMSOL called MUMPS and PARDISO, a similar result was obtained of roughly 10 hours of computation and 10Go of RAM on the same computer as the one used for CIVA. According to COMSOL's website [27], such large problem scale linearly with the number of degrees of freedom. Hence, by applying the area factor of 100, it can be deduced that the COMSOL software would require approximately 40 days of computation on the same CPU and 1000Go of RAM. This value is to be compared to the actual performance obtained with CIVA of 4 hours and 22 minutes with a 0.7 Go of RAM, hence a time improvement of a factor 200 on the same hardware. This order of mag- 

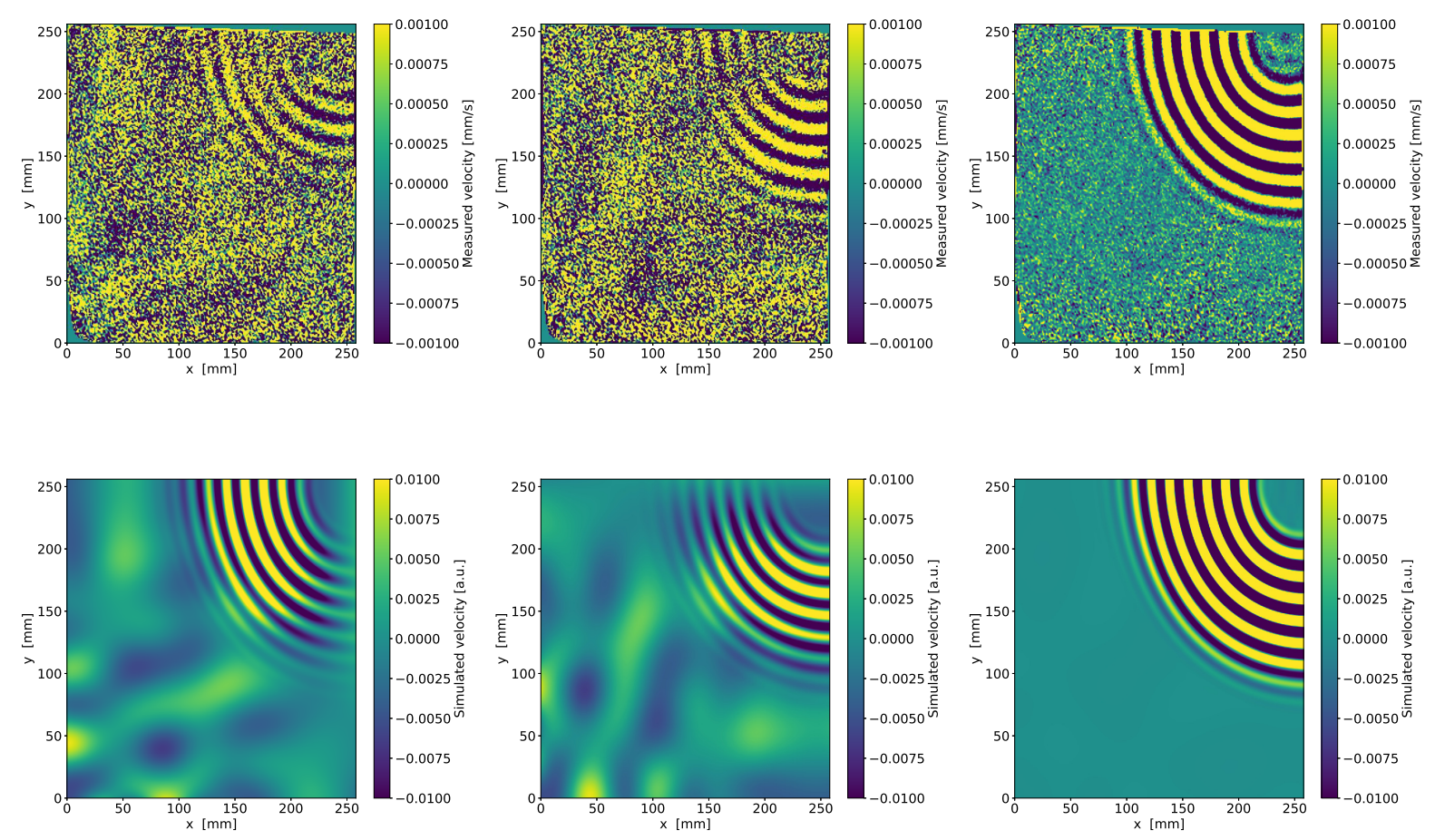

Fig. 6: Comparison of the three components of the wavefield at $\mathrm{t}=100 \mu \mathrm{s}$ at $50 \mathrm{kHz}$ : Top: experiments data, bottom: simulations; from left to right: in-plane horizontal, in-plane vertical and out-of-plane components

\begin{tabular}{|l|l|l|l|l|}
\cline { 2 - 5 } \multicolumn{1}{c|}{} & Time step & \#DoF & CPU usage & $\begin{array}{l}\text { RAM } \\
\text { usage }\end{array}$ \\
\hline $50 \mathrm{kHz}$ & $7.8 \mathrm{~ns}$ & $7 \times 10^{6}$ & $1 \mathrm{~h} 37 \mathrm{mn}$ & $0.3 \mathrm{Go}$ \\
\hline $100 \mathrm{kHz}$ & $7.8 \mathrm{~ns}$ & $19 \times 10^{6}$ & $4 \mathrm{~h} 22 \mathrm{mn}$ & 0.7 Go \\
\hline $150 \mathrm{kHz}$ & $7.3 \mathrm{~ns}$ & $36 \times 10^{6}$ & $8 \mathrm{~h} 26 \mathrm{mn}$ & $1.4 \mathrm{Go}$ \\
\hline $200 \mathrm{kHz}$ & $5.5 \mathrm{~ns}$ & $60 \times 10^{6}$ & $17 \mathrm{~h} 36 \mathrm{mn}$ & 2.6 Go \\
\hline
\end{tabular}

Table 1: Time stepping, approximate number of degrees of freedom (\#DoF) and computation performance for the wavefield simulation using CIVA on a Intel Xeon X5690@3.47GHz, 3.46GHz, 2x6 cores, 24Go RAM.

nitude is compatible with the comparison of the composite benchmark previously discussed [12] and published in [20].

\section{Validation of wave propagation in a finite composite panel}

As the wavefield analysis presented in the previous section only allows for the validation of the A0 mode to a frequency up to $100 \mathrm{kHz}$, this section focuses on another OGW dataset to extend the validation range.

\subsection{Experimental data}

The database under consideration in this section is also provided by the OGW initiative [22] and consists in a com- posite panel of similar material properties instrumented by 12 circular piezoelectric transducers of diameter $10 \mathrm{~mm}$ as depicted in figure 11. The database contains the round-robin measurement (i.e. each sensor is activated sequentially while the others are used as emitters) with 5-cycle tone bursts of central frequency from 40 to $260 \mathrm{kHz}$ every $20 \mathrm{kHz}$. Note that the distance from the sensors to the closest edge is of 3 $\mathrm{cm}$ only which means it is impossible in this dataset to observe incident wavepackets alone, as wavepackets will systematically be superimposed with the reflected wavepackets from the edges.

\subsection{Simulated configurations}

First, the pristine configurations at 40,100, 160 and 240 $\mathrm{kHz}$ are reproduced as in the OGW database for direct Ascan comparison. The piezoelectric sensors are modelled as described in Section 2.2 with a radial load defined in a ring of diameter 9 to $11 \mathrm{~mm}$. Simulations are run for $800 \mu \mathrm{s}$ at $40 \mathrm{kHz}$ and $500 \mu s$ at the other frequencies to ensure capturing the arrival of the first incident wave packet of the slowest mode (A0). The mesh refinement is automatically computed by the process described in section 2.1 for the four inspection frequencies.

\subsection{Validation results}

For the graphical comparison of the signals emitted and received by piezoelectric sensors, the path 1 to 10 is chosen arbitrarily. A more detailed analysis shows that this path ex- 

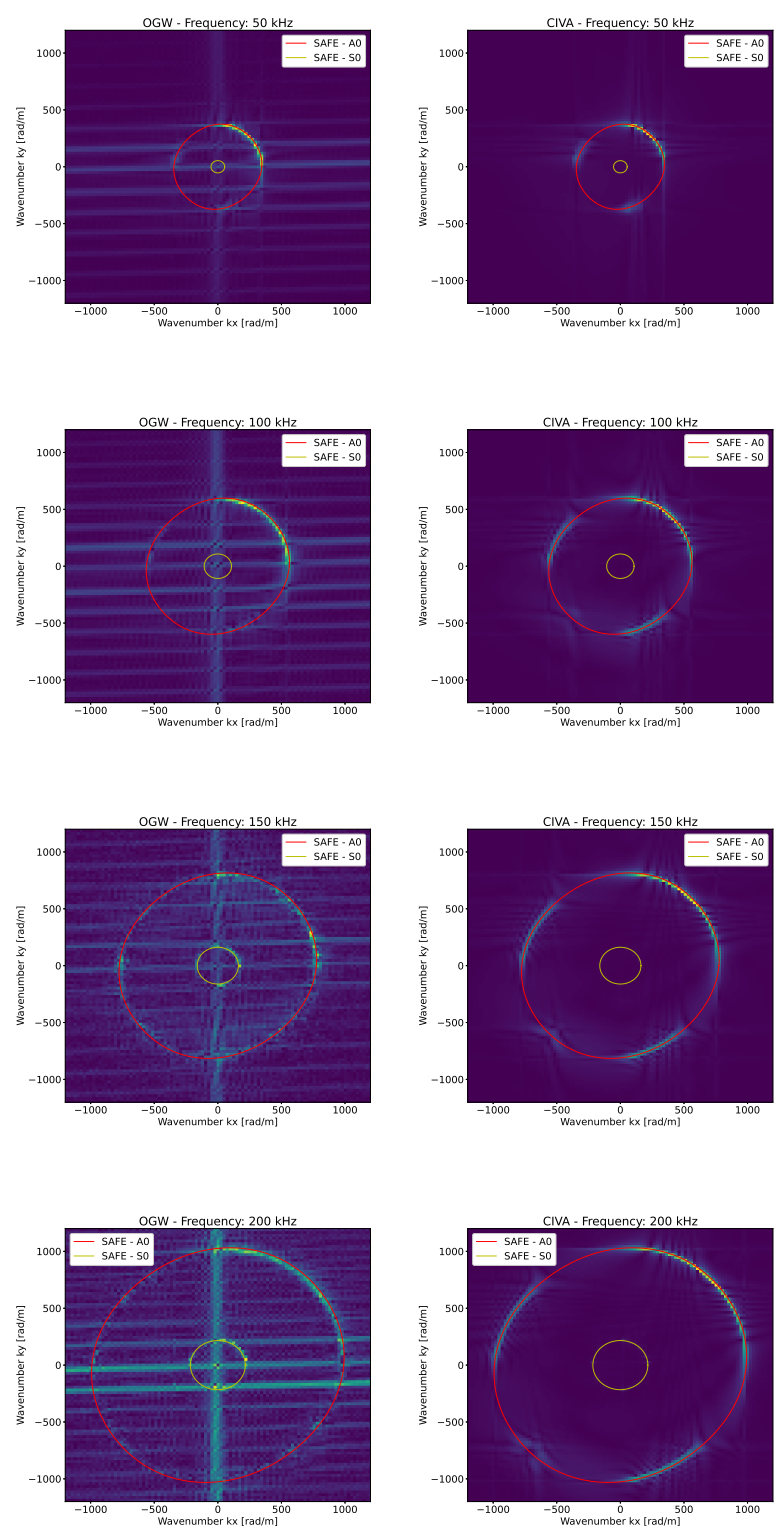

Fig. 7: Comparison of the dispersion curves of the out-ofplane component of the wavefield (left: experiments, right: simulations) from 50 (top) to $200 \mathrm{kHz}$ (bottom)

hibits comparable results with the other paths. Furthermore, due to the simplified sensor model described in section 2.2, the amplitude of the signals generated in the simulations are dimensionless, hence all the signals are normalized to unity within the time window they are represented in. Finally, to simplify the visualisation, the validation focuses first on the S0 mode in figure 12 with a truncated time window to remove the A0 mode, then the A0 mode is discussed in figure 13 with a longer time window. A computation option in CIVA allows to separate components of the signals corresponding to symmetric and antisymmetric modes. This option is used in the following, in particular to study the A0 mode without the effect of the reflected S0 mode at the same time.

\subsubsection{S0 analysis}

In figure 12, the experimental versus simulation signals are shown for the 4 frequencies of interest, with a time window focusing on the $\mathrm{S} 0$ mode (before the A0 first wavepacket). It must be reminded that the first harmonic packet in the experimental data at $t=0$ and shaded in grey, corresponds to the electronic coupling, which is not an elastic wave to be reproduced in the simulations. The first observation on these results is that the time of flight of the incident wave packet at the 4 simulation frequencies matches very well, both between experiment and simulation, but also with the theoretical time of flight value computed by the SAFE technique and represented by the yellow shaded area. It must however be noticed that at 100 and $240 \mathrm{kHz}$, the numericallycalculated incident wave-packet is separated into two nearly overlapping wave-packets, visible by the two nearby envelope maximums, while this is not the case in the experimental results. The two maximums in the simulations are the incident and its immediate reflection from the edge near the sensor, which are somewhat merged in the experiment, which may be due to a smoother reflection from the edge. Moreover, a non negligible time of flight shift is observed at 240 $\mathrm{kHz}$ : the experimental time of flight is $111 \mu \mathrm{s}$ and is outside the theoretical time of arrival, while it it measured at $107 \mu \mathrm{s}$ in simulations. The error between the two maximums is equal to one cycle at the excitation frequency $240 \mathrm{kHz}$. This effect will be further discussed in section 5.4.

The second major observation concerns the amplitude of the wave-packets. Indeed, at the two highest frequencies, within the observed time window, the maximum of the signal is due to the incident wave-packet, which is not the case at $100 \mathrm{kHz}$, due to the symmetry of the configuration and the constructive interferences from the various edges. Moreover, it must be reminded that the simulations do not include attenuation, which is why the amplitude is seen to increase in the $40 \mathrm{kHz}$ plot, while it is mostly stable in the experiment due to a balance between attenuation and constructive interferences.

\subsubsection{A0 analysis}

In figure 13, the experimental versus simulated signals are shown for the 4 frequencies of interest, with a time window focusing on the $\mathrm{A} 0$ mode. To simplify the visualization, the signals are truncated to focus on the A0 mode. The A0 simulated mode is therefore compared to the full experimental signal (i.e. A0 + S0) as modes can not be separated in the experiment. First, it must be observed that at frequencies 100, 160 and $240 \mathrm{kHz}$, no clear A0 wave-packet is visible in the experimental data within the theoretical window of arrival (yellow shaded area). This is because the A0 mode is highly attenuated at these frequencies, and therefore is largely hidden within the multiple S0 reflections. Hence, the only conclusion to be drawn from these frequencies is that the simulated first wave-packet of the A0 mode falls within the theoretical time of arrival window.

At $40 \mathrm{kHz}$ however, the $\mathrm{A} 0$ mode dominates the experimental signals and it can be observed that its time of flight 

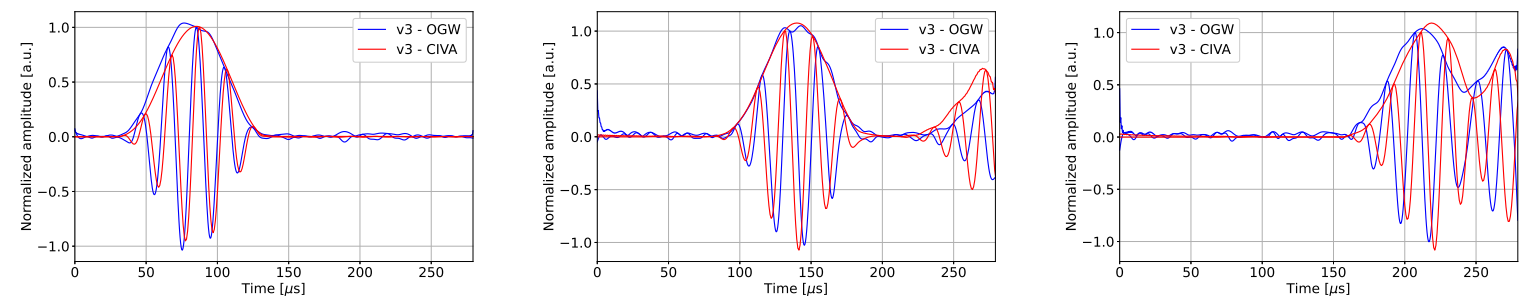

Fig. 8: Comparison of the out-of-plane velocity component v3 at $50 \mathrm{kHz}$ for positions $p_{1}=(200,200), p_{2}=(225,100)$ and $p_{3}=(100,50) \mathrm{mm}$
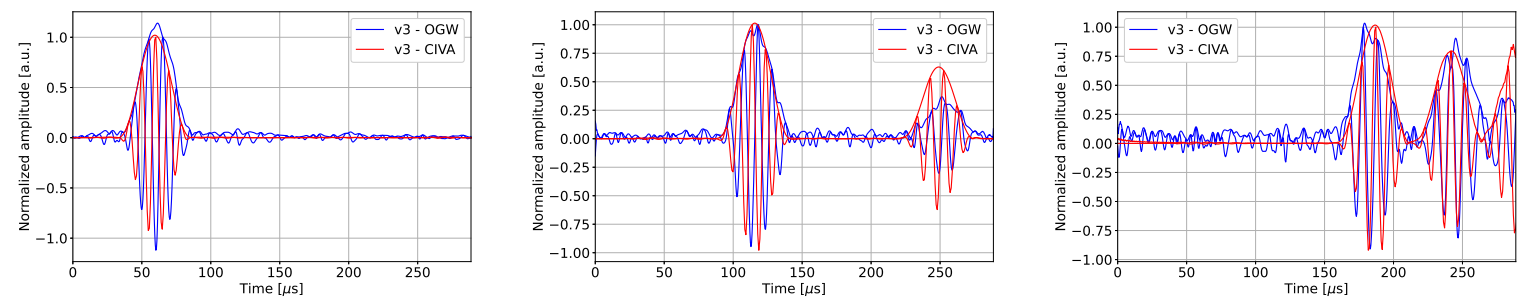

Fig. 9: Comparison of the out-of-plane velocity component v3 at $100 \mathrm{kHz}$ for positions $p_{1}=(200,200), p_{2}=(225,100)$ and $p_{3}=(100,50) \mathrm{mm}$
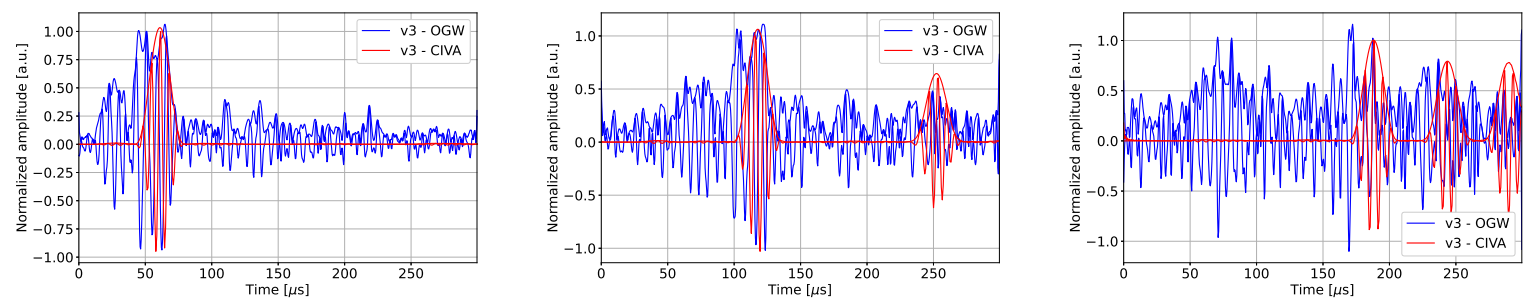

Fig. 10: Comparison of the out-of-plane velocity component v3 at $150 \mathrm{kHz}$ for positions $p_{1}=(200,200), p_{2}=(225,100)$ and $p_{3}=(100,50) \mathrm{mm}$

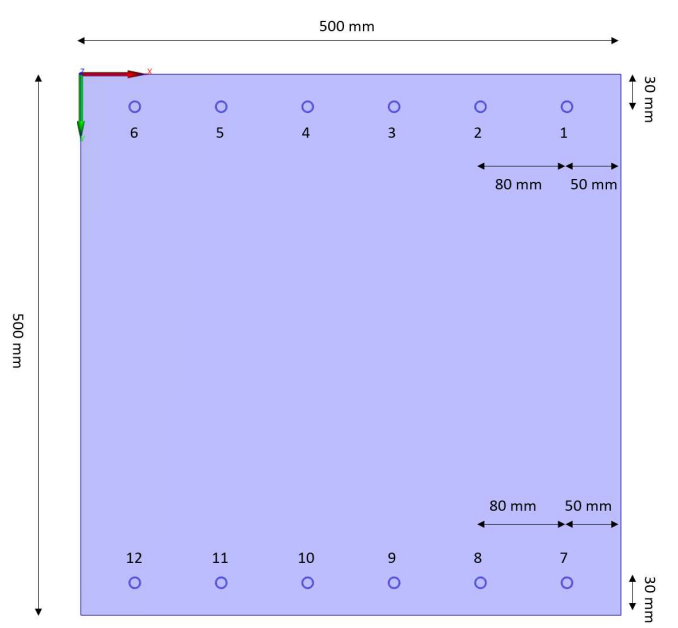

Fig. 11: Schematic of the configuration of the composite panel with 12 piezoelectric sensors does not match the simulated one, which does not arrive within the theoretical time of arrival window. The experimental time of flight, measured as the maximum of the envelope, is $476 \mu \mathrm{s}$ while it is measured at $501 \mu \mathrm{s}$ in the simulation, which is a significant shift, equal to roughly one cycle at the excitation frequency $40 \mathrm{kHz}$. This effect will be further discussed in the incoming section.

\subsection{Discussion}

A non-negligible time shift of approximately one period of the central frequency of the excitation was observed for the A0 mode at $40 \mathrm{kHz}$ and for the $\mathrm{S} 0$ mode at $240 \mathrm{kHz}$. It is thought that this shift is majorly due to a different behaviour of the edges of the plate between the model and the experiment. To demonstrate this, another simulation was conducted on a larger $700 \times 700 \times 2 \mathrm{~mm}^{3}$ plate, while all the other parameters remain the same. This is equivalent to having an infinite plate for the A0 mode within the time window and the path under consideration at $40 \mathrm{kHz}$. The result 

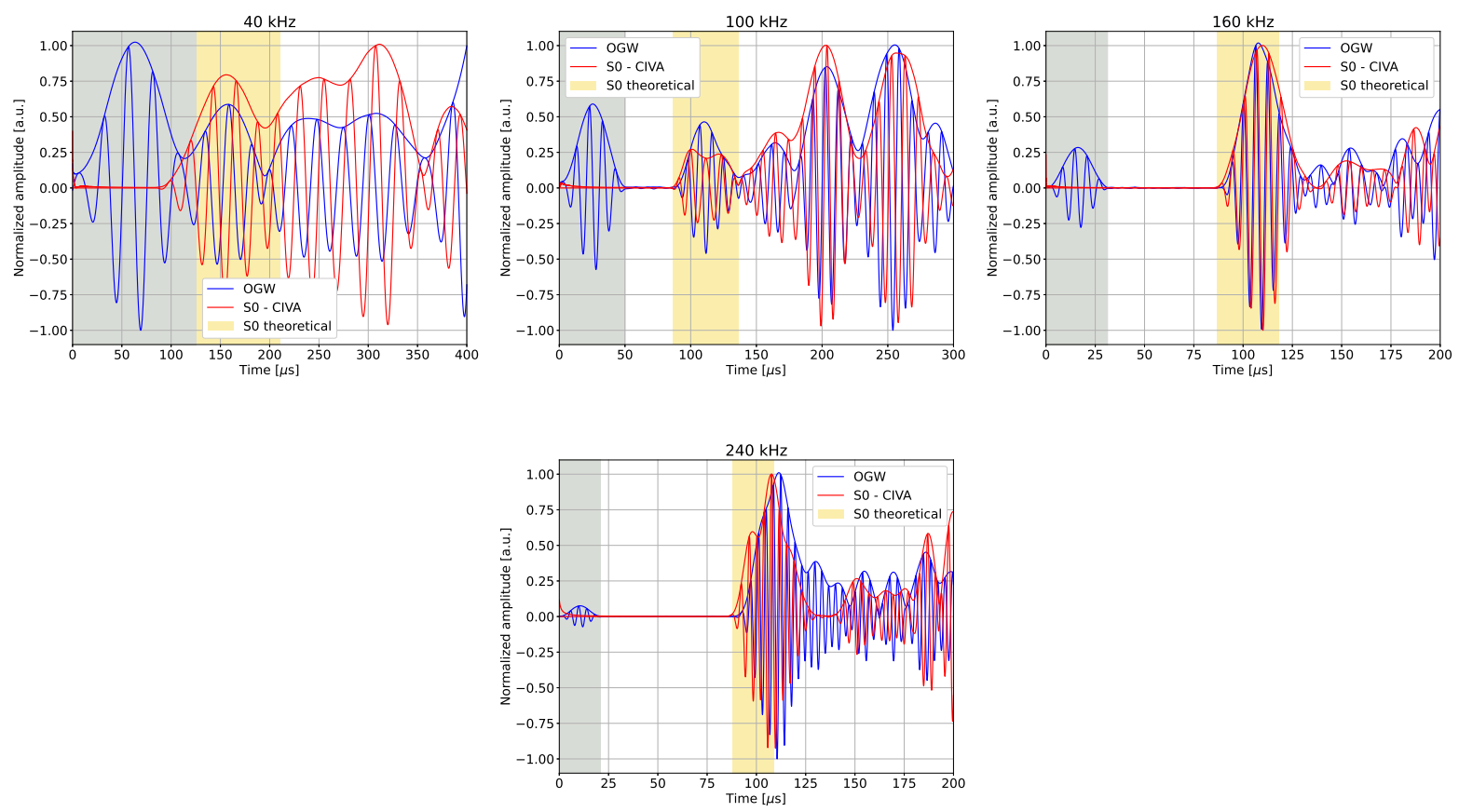

Fig. 12: Comparison of the signal received by sensor 10 while sensor 1 acts as emitter at 40, 100, 160 and $240 \mathrm{kHz}$ focusing on the S0 mode (blue: OGW, red: CIVA S0 mode). The grey shaded area corresponds to the electronic coupling while the yellow area is the theoretical time of arrival window of the incident $\mathrm{S} 0$ wave-packet, as computed by SAFE and neglecting dispersion.
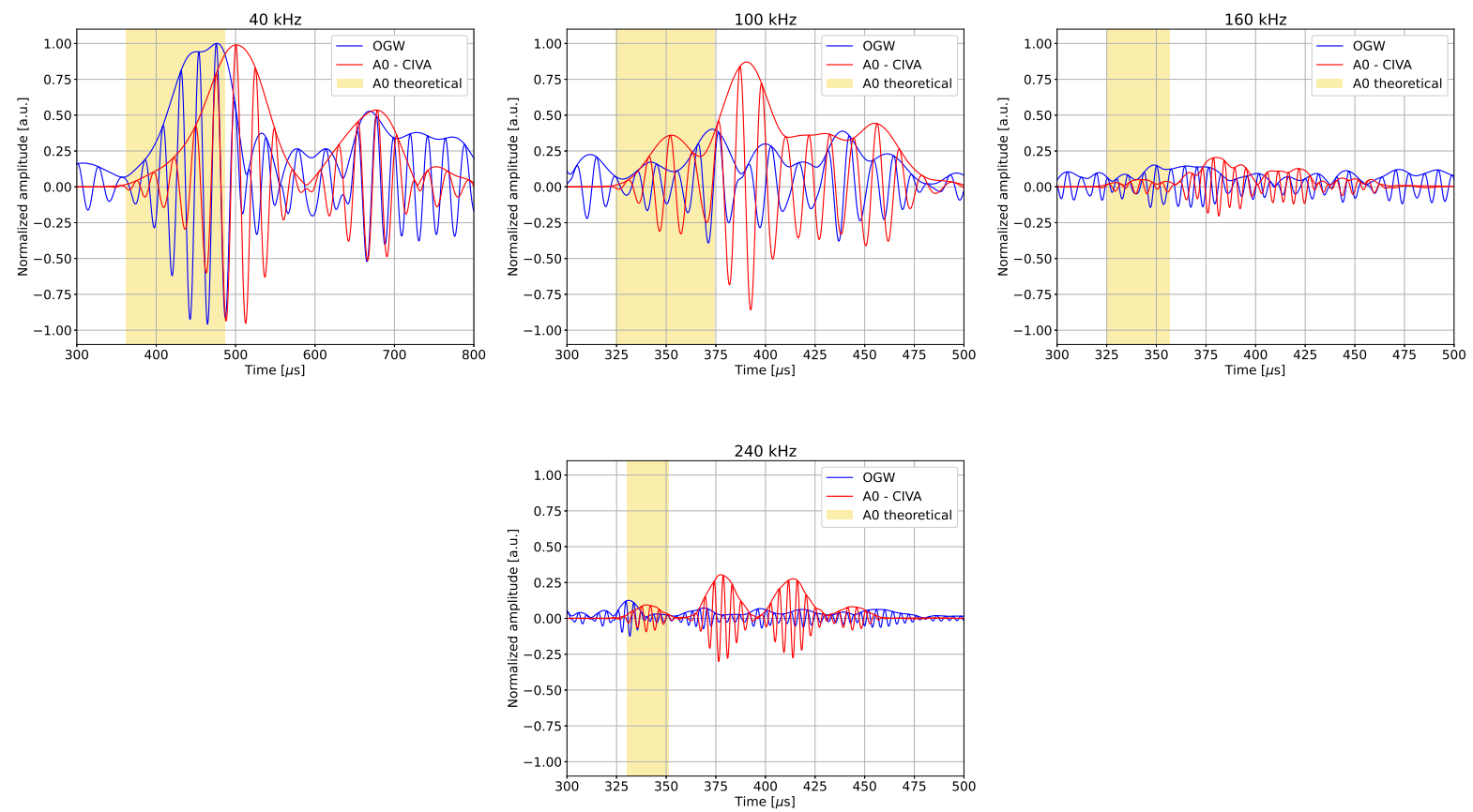

Fig. 13: Comparison of the signal received by sensor 10 while sensor 1 acts as emitter at 40, 100, 160 and $240 \mathrm{kHz}$ focusing on the A0 mode (blue: OGW, red: CIVA S0 mode). The grey shaded area corresponds to the electronic coupling while the yellow area is the theoretical time of arrival window of the incident S0 wave-packet, as computed by SAFE and neglecting dispersion. 


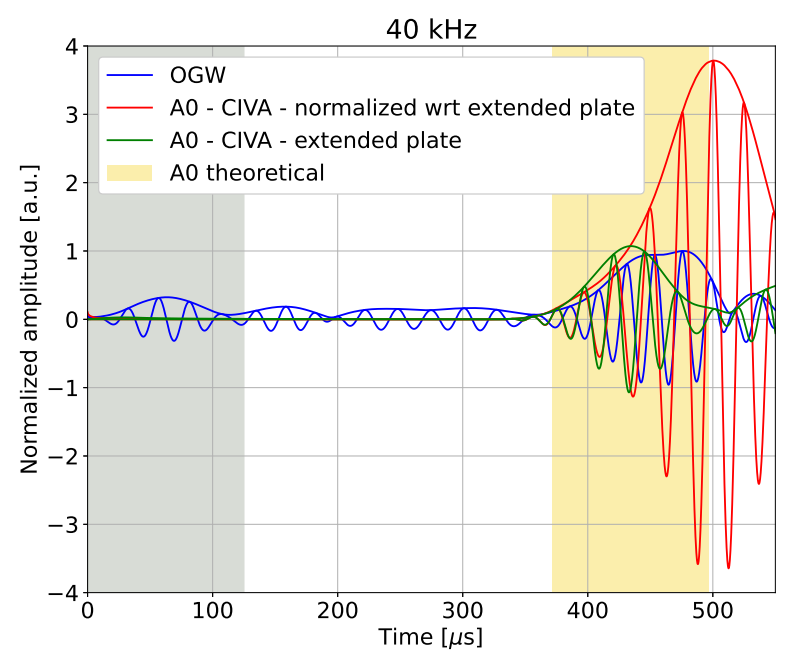

Fig. 14: Comparison of the signal received by sensor 10 while sensor 1 acts as emitter at $40 \mathrm{kHz}$ (blue: OGW, red: CIVA on the finite plate and green : CIVA in the extended plate)

of the A0 mode as received by the sensor 10 in this infinite plate is represented in green in the figure 14, as well as the simulated signal in the finite plate (i.e. result presented in figure 13) and the experimental signal. Note that in this picture, both simulated signals are normalized with respect to the maximum of the amplitude of the simulated signal in the extended panel. Two major effects can be observed after removing the effect of the edges: the time of flight falls perfectly within the theoretical time window of arrival and the maximum amplitude is decreased by a factor of 4 with respect to the one in the finite simulated panel. This result is in accordance with the expectation: in the finite plate, in addition to the direct path from sensor 1 to 10,3 other paths with a single edge reflection have similar time of flights and combine constructively to create an amplitude of 4 . This explains both the difference of time of flight and amplitude between the simulations. Moreover, the experimental time of flight lands half-way between the signals from the finite and the extended plates. Our interpretation is that the edges do have an influence on the experimentally measured signals, but the reflections are neither fully constructive nor destructive. This difference in the interaction of the reflections may come from higher uncertainties regarding the edges of plates, which may not be well taken into account in the simulations.

Interestingly enough, according to the dispersion curves of the composite panel, the effect described in this section is observed when the $\mathrm{S} 0$ wavelength at $240 \mathrm{kHz}$ is equal to 24 $\mathrm{mm}$ while the one of the $\mathrm{A} 0$ mode at $40 \mathrm{kHz}$ is $19 \mathrm{~mm}$, which correspond roughly to twice the back-and-forth distance between the edge and the actuator. This effect might therefore be interpreted by some sort of resonance mechanism between the edge of the plate and the actuator.
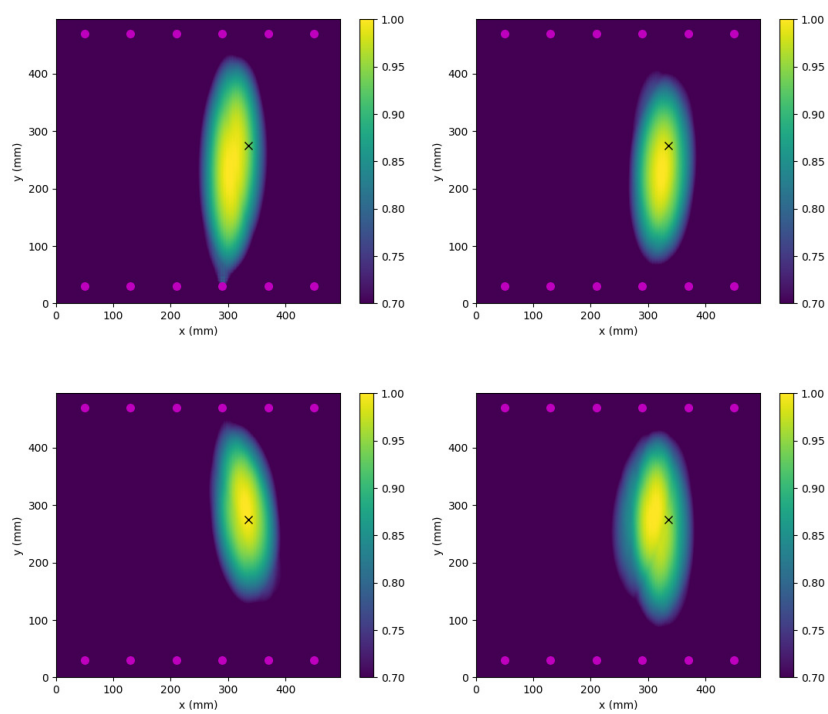

Fig. 15: Images obtained using RAPID on experimental (left) and simulated (right) data for defect D14 (black cross). Top row: center frequency of $40 \mathrm{kHz}$. Bottom row: center frequency of $100 \mathrm{kHz}$.

\subsection{Defect imaging}

The same OGW database also contains scans with an added mass to simulate a defect at 28 positions. However, if this type of defect is often used in the laboratory to avoid permanently damaging the specimens, it is not necessary to go to such lengths in simulations as holes and delaminations can be created at will. For this reason and for qualitative comparison of the defect detection, simulations with defects were conducted in CIVA, in which the defect was modelled by a circular through-hole of the same diameter as the added mass. The imaging was performed using the reconstruction algorithm for probabilistic inspection of defects [28] using the same parameters as in [22], that is $\beta=1.1$ and using only datasets corresponding to sensors located on opposite sides. Results are shown in figure 15 for two central frequencies, for both the simulated and experimental data. There is a good match between imaging results in both cases, showing the interest of the simulation for the study and design of an SHM system.This results shows that representative and useful SHM data can be generated albeit the minor data mismatch, principally because the mismatch is mitigated by the use of a baseline in the imaging algorithm.

\section{Validation of wave propagation in a stiffened compos- ite panel}

Finally, stiffened panels were considered using the same experimental protocol and sensor configurations in OGW [29]. Those configurations were simulated in CIVA and are depicted in figure 16 . 

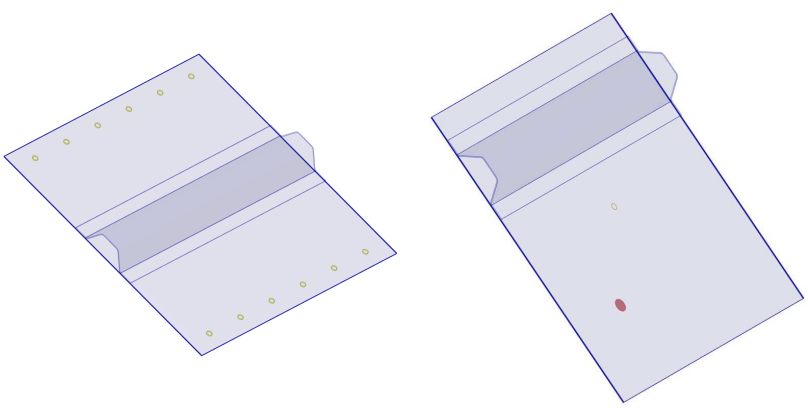

Fig. 16: View of the stiffened panel in CIVA, SHM plate case (left) and wavefield case (right). Defect (delamination) in red, sensors in yellow.
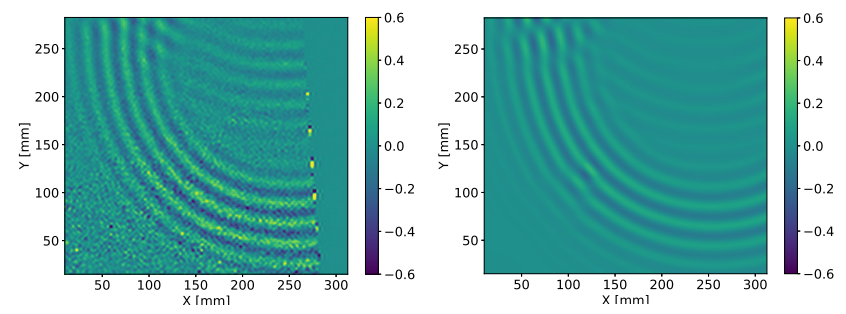

Fig. 17: Snapshots of the wavefield scan for experimental (left) and simulated (right) data, out-of plane displacement component at $\mathrm{t}=200 \mu \mathrm{s}$.

\subsection{Wavefield plate}

First, the wavefield case is considered and a simulation for a center frequency of $40 \mathrm{kHz}$ is performed. The wavefield is extracted on the bottom-left quarter of the plate as in the data available in OGW. Note that a defect is simulated in this part of the plate, using an added mass of radius 10 $\mathrm{mm}$ in OGW and a circular delamination at a depth of 0.875 $\mathrm{mm}$ and of the same size in CIVA (delamination is obtained by disconnecting the adjacent 3D solid elements). Snapshots of the resulting wavefield are plotted in figure 17. A good qualitative agreement is seen between simulation and experiment. In particular, a wave-packet travelling back from the stiffener (i.e. the wave propagating from top to bottom in the upper half), is seen. The wave front's position is the same in both cases, but its relative amplitude is smaller in the simulation. This may come from the already mentioned simple model used for the edges of plates and here of the stiffener, or from uncertainties on the geometry of the stiffener. The effect of the defect on the wave's propagation can be seen in the simulated wavefield at $\mathrm{t}=100 \mu \mathrm{s}$ but not in the experimental one. The Ascan comparison is not shown in this case, as the previous comparisons apply: for wave packets which did not cross the stiffener, a good match is observed for the time of flight but not the phase, as already observed in section 4, and for wave packets which were reflected by the stiffener there is a good match for the time of flight but not the phase nor the relative amplitude because of the lack of attenuation and the aforementioned uncertainties on the stiffener.

\subsection{SHM plate}

To conclude this section, simulations were performed for the stiffened panel with 12 piezoelectric sensors for a center frequency of $40 \mathrm{kHz}$. As was already mentioned in the case without stiffener, a mismatch exists between the simulation and the experiment because of the edges of the plate, which hinder the comparison. To circumvent this difficulty, Ascans for three emitter-receiver couples with and without the stiffener are shown in figure 18. The same effect of the stiffener is seen in both cases, that is a loss in amplitude of the S0 mode and a small delay. Wave packets at later times are harder to compare because of the reflections on the edges of the plate.

\section{Conclusions}

This paper focuses on the validation of the guided wave propagation simulated by CIVA for SHM in three use-cases: a stainless steel pipe, a flat composite panel and a stiffened composite panel. For the first use-case, the elastic properties were empirically calibrated while for the other two, the properties were carefully measured by the data providers. Good match between experiments and simulations were observed in the three cases. All the mismatches observed between simulations and experiments are attributed to unknown parameters: thickness variation in the pipe, edge reflections in the composite panel and stiffener geometry and coupling. The computational performance of CIVA was compared with COMSOL and a significant improvement was shown on the same hardware.

Subsequent validation work will focus on the validation of the transduction of the sensor and the response from defects. Future versions of CIVA for SHM will include attenuation and more accurate sensor models, and will lift the meshing constraints to handle arbitrary geometries.

\section{References}

[1] Kulakovskyi, A., Mesnil, O., Chapuis, B., d'Almeida, O., and Lhemery, A. "Statistical analysis of guided wave imaging algorithms performance illustrated by a simple shm configuration [under publication]".

[2] Druet, T., Tastet, J.-L., Chapuis, B., and Moulin, E., 2019. "Autocalibration method for guided wave tomography with undersampled data". Wave Motion, 89, pp. 265-283.

[3] Baronian, V., Bourgeois, L., Chapuis, B., and Recoquillay, A., 2018. "Linear sampling method applied to non destructive testing of an elastic waveguide: theory, numerics and experiments". Inverse Problems, 34(7), p. 075006.

[4] Miorelli, R., Fisher, C., Kulakovskyi, A., Mesnil, O., Chapuis, B., and d'Almeida, O. "Defect sizing in guided wave imaging structural health monitoring using convolutional neural networks [under publication]".

[5] Bartoli, I., Marzani, A., Di Scalea, F. L., and Viola, E., 2006. "Modeling wave propagation in damped waveg- 

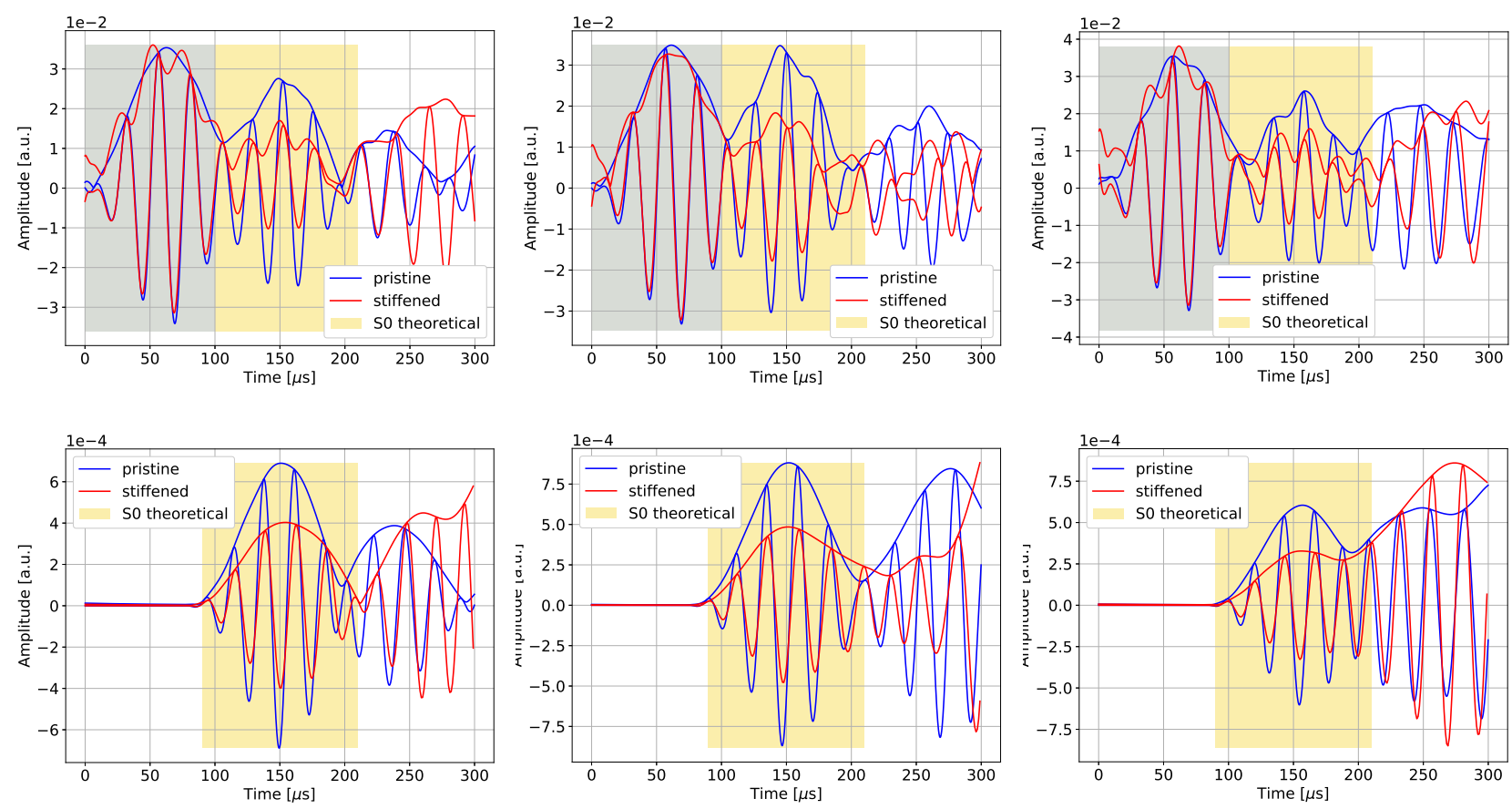

Fig. 18: Ascans of the SHM plate from experiment (top) and simulation (bottom) for sensor 3 as emitter and respectively sensor 7, 10 and 12 as receiver for the left, middle and right plot. Blue: signals without stiffener. Red: signals with stiffener. Grey area: sensor's coupling.

uides of arbitrary cross-section". Journal of sound and vibration, 295(3-5), pp. 685-707.

[6] Treyssède, F., and Laguerre, L., 2013. "Numerical and analytical calculation of modal excitability for elastic wave generation in lossy waveguides". The Journal of the Acoustical Society of America, 133(6), pp. 38273837.

[7] Barras, J., Lhémery, A., and Impériale, A., 2020. "Modal pencil method for the radiation of guided wave fields in finite isotropic plates validated by a transient spectral finite element method". Ultrasonics, 103, p. 106078.

[8] Gopalakrishnan, S., Chakraborty, A., and Mahapatra, D. R., 2007. Spectral finite element method: wave propagation, diagnostics and control in anisotropic and inhomogeneous structures. Springer Science \& Business Media.

[9] Baronian, V., Lhémery, A., and Jezzine, K., 2011. "Hybrid safe/fe simulation of inspections of elastic waveguides containing several local discontinuities or defects". In AIP Conference Proceedings, Vol. 1335, American Institute of Physics, pp. 183-190.

[10] Benmeddour, F., Treyssède, F., and Laguerre, L., 2011. "Numerical modeling of guided wave interaction with non-axisymmetric cracks in elastic cylinders". International journal of Solids and Structures, 48(5), pp. 764774.

[11] Mesnil, O., Imperiale, A., Demaldent, E., Baronian, V., and Chapuis, B., 2018. "Simulation tools for guided wave based structural health monitoring". In AIP
Conference Proceedings, Vol. 1949, AIP Publishing, p. 050001.

[12] Leckey, C. A., Wheeler, K. R., Hafiychuk, V. N., Hafiychuk, H., and Timuçin, D. A., 2018. "Simulation of guided-wave ultrasound propagation in composite laminates: Benchmark comparisons of numerical codes and experiment". Ultrasonics, 84, pp. 187-200.

[13] Huthwaite, P., 2014. "Accelerated finite element elastodynamic simulations using the gpu". Journal of Computational Physics, 257, pp. 687-707.

[14] Shen, Y., and Cesnik, C. E., 2015. "Hybrid local fem/global lisa modeling of guided wave propagation and interaction with damage in composite structures". In Health Monitoring of Structural and Biological Systems 2015, Vol. 9438, International Society for Optics and Photonics, p. 94380J.

[15] Kudela, P., Moll, J., and Fiborek, P., 2020. "Parallel spectral element method for guided wave based structural health monitoring". Smart Materials and Structures, 29(9), p. 095010.

[16] Gregory, E., 2020. "Gpu accelerated simulation for ut inspection of aerospace materials". NDT in Aerospace.

[17] Ostachowicz, W., Kudela, P., Krawczuk, M., and Zak, A., 2011. Guided waves in structures for SHM: the time-domain spectral element method. John Wiley \& Sons.

[18] Kudela, P., 2016. "Parallel implementation of spectral element method for lamb wave propagation modeling". International Journal for Numerical Methods in Engineering, 106(6), pp. 413-429. 
[19] Imperiale, A., and Demaldent, E., 2019. "A macroelement strategy based upon spectral finite elements and mortar elements for transient wave propagation modeling. application to ultrasonic testing of laminate composite materials". International Journal for $\mathrm{Nu}$ merical Methods in Engineering, 119(10), pp. 964 990.

[20] Mesnil, O., Imperiale, A., Demaldent, E., and Chapuis, B., 2019. "Validation of spectral finite element simulation tools dedicated to guided wave based structure health monitoring". Vol. 2102, p. 050018.

[21] EXTENDE, 2020. http://www.extende.com.

[22] Moll, J., Kathol, J., Fritzen, C.-P., Moix-Bonet, M., Rennoch, M., Koerdt, M., Herrmann, A. S., Sause, M. G., and Bach, M., 2019. “Open guided waves: online platform for ultrasonic guided wave measurements". Structural Health Monitoring, 18(56), pp. 1903-1914.

[23] De Basabe, J. D., and Sen, M. K., 2007. "Grid dispersion and stability criteria of some common finiteelement methods for acoustic and elastic wave equations". Geophysics, 72(6), pp. T81-T95.

[24] Imperiale, A., Leymarie, N., and Demaldent, E., 2020. "Numerical modeling of wave propagation in anisotropic viscoelastic laminated materials in transient regime: Application to modeling ultrasonic testing of composite structures". International Journal for $\mathrm{Nu}$ merical Methods in Engineering, 121(15), pp. 33003338.

[25] Crawley, E. F., and De Luis, J., 1987. "Use of piezoelectric actuators as elements of intelligent structures". AIAA journal, 25(10), pp. 1373-1385.

[26] Marlett, K., Ng, Y., and Tomblin, J., 2011. "Hexcel $8552 \mathrm{im} 7$ unidirectional prepreg $190 \mathrm{gsm} \& 35 \% \mathrm{rc}$ qualification material property data report". National Center for Advanced Materials Performance, Wichita, Kansas. Test Report CAM-RP-2009-015, Rev. A, pp. 1238.

[27] Comsol, 2020. How much memory is needed to solve large comsol models?

[28] Hay, T., Royer, R., Gao, H., Zhao, X., and Rose, J. L., 2006. "A comparison of embedded sensor lamb wave ultrasonic tomography approaches for material loss detection". Smart materials and structures, 15(4), p. 946.

[29] Moll, J., Kexel, C., Kathol, J., Fritzen, C.-P., MoixBonet, M., Willberg, C., Rennoch, M., Koerdt, M., and Herrmann, A., 2020. "Guided waves for damage detection in complex composite structures: The influence of omega stringer and different reference damage size". Applied Sciences, 10(9), p. 3068. 